\title{
eV-Scale Sterile Neutrino Search Using Eight Years of Atmospheric Muon Neutrino Data from the IceCube Neutrino Observatory
}

M. G. Aartsen, ${ }^{17}$ R. Abbasi, ${ }^{16}$ M. Ackermann, ${ }^{56}$ J. Adams, ${ }^{17}$ J. A. Aguilar, ${ }^{12}$ M. Ahlers, ${ }^{21}$ M. Ahrens, ${ }^{47}$ C. Alispach, ${ }^{27}$ N. M. Amin,${ }^{40}$ K. Andeen, ${ }^{38}$ T. Anderson, ${ }^{53}$ I. Ansseau, ${ }^{12}$ G. Anton, ${ }^{25}$ C. Argüelles $\odot,{ }^{14}$ J. Auffenberg, ${ }^{1}$ S. Axani, ${ }^{14}$ H. Bagherpour, ${ }^{17}$ X. Bai,${ }^{44}$ A. Balagopal,${ }^{30}$ A. Barbano, ${ }^{27}$ S. W. Barwick,${ }^{29}$ B. Bastian,${ }^{56}$ V. Basu, ${ }^{36}$ V. Baum, ${ }^{37}$ S. Baur, ${ }^{12}$ R. Bay, ${ }^{8}$ J. J. Beatty, ${ }^{19,20}$ K.-H. Becker, ${ }^{55}$ J. Becker Tjus, ${ }^{11}$ S. BenZvi, ${ }^{46}$ D. Berley, ${ }^{18}$ E. Bernardini, ${ }^{56,{ }^{*}}$ D. Z. Besson, ${ }^{31,+}$ G. Binder, ${ }^{8,9}$ D. Bindig, ${ }^{55}$ E. Blaufuss, ${ }^{18}$ S. Blot, ${ }^{56}$ C. Bohm, ${ }^{47}$ S. Böser, ${ }^{37}$ O. Botner, ${ }^{54}$ J. Böttcher, ${ }^{1}$ E. Bourbeau, ${ }^{21}$ J. Bourbeau, ${ }^{36}$ F. Bradascio, ${ }^{56}$ J. Braun, ${ }^{36}$ S. Bron, ${ }^{27}$ J. Brostean-Kaiser, ${ }^{56}$ A. Burgman, ${ }^{54}$ J. Buscher, ${ }^{1}$ R. S. Busse, ${ }^{39}$

T. Carver, ${ }^{27}$ C. Chen, ${ }^{6}$ E. Cheung, ${ }^{18}$ D. Chirkin, ${ }^{36}$ S. Choi ${ }^{49}$ B. A. Clark, ${ }^{23}$ K. Clark, ${ }^{32}$ L. Classen, ${ }^{39}$ A. Coleman ${ }^{40}$ G. H. Collin, ${ }^{14}$ J. M. Conrad, ${ }^{14}$ P. Coppin, ${ }^{13}$ P. Correa, ${ }^{13}$ D. F. Cowen,${ }^{52,53}$ R. Cross,${ }^{46}$ P. Dave, ${ }^{6}$ C. De Clercq, ${ }^{13}$ J. J. DeLaunay, ${ }^{53}$ H. Dembinski, ${ }^{40}$ K. Deoskar, ${ }^{47}$ S. De Ridder ${ }^{28}$ A. Desai,${ }^{36}$ P. Desiati, ${ }^{36}$ K. D. de Vries, ${ }^{13}$ G. de Wasseige, ${ }^{13}$ M. de With, ${ }^{10}$ T. DeYoung, ${ }^{23}$ S. Dharani, ${ }^{1}$ A. Diaz, ${ }^{14}$ J. C. Díaz-Vélez ${ }^{36}$ H. Dujmovic,${ }^{30}$ M. Dunkman,${ }^{53}$ M. A. DuVernois, ${ }^{36}$ E. Dvorak, ${ }^{44}$ T. Ehrhardt,${ }^{37}$ P. Eller, ${ }^{53}$ R. Engel,${ }^{30}$ P. A. Evenson, ${ }^{40}$ S. Fahey,${ }^{36}$ A. R. Fazely, ${ }^{7}$ A. Fedynitch, ${ }^{57}$ J. Felde, ${ }^{18}$ A. T. Fienberg, ${ }^{52}$ K. Filimonov, ${ }^{8}$ C. Finley, ${ }^{47}$ D. Fox ${ }^{52}$ A. Franckowiak, ${ }^{56}$ E. Friedman, ${ }^{18}$ A. Fritz, ${ }^{37}$ T. K. Gaisser, ${ }^{40}$ J. Gallagher, ${ }^{35}$ E. Ganster, ${ }^{1}$ S. Garrappa ${ }^{56}$ L. Gerhardt, ${ }^{9}$ T. Glauch, ${ }^{26}$ T. Glüsenkamp, ${ }^{25}$ A. Goldschmidt, ${ }^{9}$ J. G. Gonzalez, ${ }^{40}$ D. Grant, ${ }^{23}$ T. Grégoire, ${ }^{53}$ Z. Griffith, ${ }^{36}$ S. Griswold, ${ }^{46}$ M. Günder, ${ }^{1}$ M. Gündüz, ${ }^{11}$ C. Haack, ${ }^{1}$ A. Hallgren, ${ }^{54}$ R. Halliday, ${ }^{23}$ L. Halve,${ }^{1}$ F. Halzen, ${ }^{36}$ K. Hanson, ${ }^{36}$ J. Hardin ${ }^{36}$ A. Haungs ${ }^{30}$ S. Hauser, ${ }^{1}$ D. Hebecker, ${ }^{10}$ D. Heereman, ${ }^{12}$ P. Heix, ${ }^{1}$ K. Helbing, ${ }^{55}$ R. Hellauer, ${ }^{18}$ F. Henningsen, ${ }^{26}$ S. Hickford ${ }^{55}$ J. Hignight, ${ }^{24}$ G. C. Hill, ${ }^{2}$ K. D. Hoffman, ${ }^{18}$ R. Hoffmann, ${ }^{55}$ T. Hoinka, ${ }^{22}$ B. Hokanson-Fasig, ${ }^{36}$ K. Hoshina, ${ }^{36, *}$ F. Huang, ${ }^{53}$ M. Huber, ${ }^{26}$ T. Huber ${ }^{30,56}$ K. Hultqvist, ${ }^{47}$ M. Hünnefeld, ${ }^{22}$ R. Hussain, ${ }^{36}$ S. In, ${ }^{49}$ N. Iovine, ${ }^{12}$ A. Ishihara, ${ }^{15}$ M. Jansson, ${ }^{47}$ G. S. Japaridze, ${ }^{5}$ M. Jeong, ${ }^{49}$ B. J. P. Jones, ${ }^{4}$ F. Jonske, ${ }^{1}$ R. Joppe, ${ }^{1}$ D. Kang, ${ }^{30}$ W. Kang, ${ }^{49}$ A. Kappes, ${ }^{39}$ D. Kappesser, ${ }^{37}$ T. Karg, ${ }^{56}$ M. Karl, ${ }^{26}$ A. Karle, ${ }^{36}$ U. Katz, ${ }^{25}$ M. Kauer, ${ }^{36}$ M. Kellermann, ${ }^{1}$ J. L. Kelley, ${ }^{36}$ A. Kheirandish, ${ }^{53}$ J. Kim, ${ }^{49}$

T. Kintscher, ${ }^{56}$ J. Kiryluk, ${ }^{48}$ T. Kittler, ${ }^{25}$ S. R. Klein, ${ }^{8,9}$ R. Koirala, ${ }^{40}$ H. Kolanoski, ${ }^{10}$ L. Köpke, ${ }^{37}$ C. Kopper, ${ }^{23}$ S. Kopper, ${ }^{51}$ D. J. Koskinen, ${ }^{21}$ P. Koundal, ${ }^{30}$ M. Kowalski, ${ }^{10,56}$ K. Krings, ${ }^{26}$ G. Krückl,${ }^{37}$ N. Kulacz, ${ }^{24}$ N. Kurahashi, ${ }^{43}$ A. Kyriacou, ${ }^{2}$ J. L. Lanfranchi, ${ }^{53}$ M. J. Larson, ${ }^{18}$ F. Lauber, ${ }^{55}$ J. P. Lazar, ${ }^{36}$ K. Leonard, ${ }^{36}$ A. Leszczyńska, ${ }^{30}$ Y. Li, ${ }^{53}$ Q. R. Liu, ${ }^{36}$ E. Lohfink, ${ }^{37}$ C. J. Lozano Mariscal,${ }^{39}$ L. Lu, ${ }^{15}$ F. Lucarelli, ${ }^{27}$ A. Ludwig, ${ }^{33}$ J. Lünemann, ${ }^{13}$ W. Luszczak, ${ }^{36}$ Y. Lyu, ${ }^{8,9}$ W. Y. Ma ${ }^{56}$ J. Madsen, ${ }^{45}$ G. Maggi, ${ }^{13}$ K. B. M. Mahn, ${ }^{23}$ Y. Makino, ${ }^{36}$ P. Mallik, ${ }^{1}$ S. Mancina ${ }^{36}$ I. C. Mariş, ${ }^{12}$

R. Maruyama, ${ }^{41}$ K. Mase, ${ }^{15}$ R. Maunu, ${ }^{18}$ F. McNally,${ }^{34}$ K. Meagher, ${ }^{36}$ M. Medici, ${ }^{21}$ A. Medina,${ }^{20}$ M. Meier, ${ }^{22}$

S. Meighen-Berger, ${ }^{26}$ J. Merz, ${ }^{1}$ T. Meures, ${ }^{12}$ J. Micallef, ${ }^{23}$ D. Mockler, ${ }^{12}$ G. Momenté, ${ }^{37}$ T. Montaruli, ${ }^{27}$ R. W. Moore, ${ }^{24}$ R. Morse, ${ }^{36}$ M. Moulai, ${ }^{14}$ P. Muth, ${ }^{1}$ R. Nagai, ${ }^{15}$ U. Naumann, ${ }^{55}$ G. Neer, ${ }^{23}$ L. V. Nguyen, ${ }^{23}$ H. Niederhausen, ${ }^{26}$ M. U. Nisa, ${ }^{23}$ S. C. Nowicki, ${ }^{23}$ D. R. Nygren, ${ }^{9}$ A. Obertacke Pollmann, ${ }^{55}$ M. Oehler, ${ }^{30}$ A. Olivas, ${ }^{18}$ A. O'Murchadha, ${ }^{12}$ E. O'Sullivan, ${ }^{47}$ T. Palczewski, ${ }^{89}$ H. Pandya, ${ }^{40}$ D. V. Pankova, ${ }^{53}$ N. Park,${ }^{36}$ G. K. Parker, ${ }^{4}$ E. N. Paudel,${ }^{40}$ P. Peiffer,${ }^{37}$ C. Pérez de los Heros, ${ }^{54}$ S. Philippen, ${ }^{1}$ D. Pieloth ${ }^{22}$ S. Pieper, ${ }^{55}$ E. Pinat,${ }^{12}$ A. Pizzuto, ${ }^{36}$ M. Plum,${ }^{38}$ Y. Popovych, ${ }^{1}$ A. Porcelli, ${ }^{28}$

M. Prado Rodriguez, ${ }^{36}$ P. B. Price, ${ }^{8}$ G. T. Przybylski, ${ }^{9}$ C. Raab, ${ }^{12}$ A. Raissi,${ }^{17}$ M. Rameez, ${ }^{21}$ L. Rauch,${ }^{56}$ K. Rawlins, ${ }^{3}$

I. C. Rea, ${ }^{26}$ A. Rehman, ${ }^{40}$ R. Reimann, ${ }^{1}$ B. Relethford,${ }^{43}$ M. Renschler, ${ }^{30}$ G. Renzi, ${ }^{12}$ E. Resconi, ${ }^{26}$ W. Rhode, ${ }^{22}$ M. Richman, ${ }^{43}$ B. Riedel, ${ }^{36}$ S. Robertson, ${ }^{9}$ M. Rongen, ${ }^{1}$ C. Rott, ${ }^{49}$ T. Ruhe,${ }^{22}$ D. Ryckbosch, ${ }^{28}$ D. Rysewyk Cantu, ${ }^{23}$ I. Safa, ${ }^{36}$ S. E. Sanchez Herrera, ${ }^{23}$ A. Sandrock, ${ }^{22}$ J. Sandroos, ${ }^{37}$ M. Santander, ${ }^{51}$ S. Sarkar, ${ }^{42}$ S. Sarkar, ${ }^{24}$ K. Satalecka, ${ }^{56}$ M. Scharf, ${ }^{1}$ M. Schaufel, ${ }^{1}$ H. Schieler, ${ }^{30}$ P. Schlunder, ${ }^{22}$ T. Schmidt, ${ }^{18}$ A. Schneider, ${ }^{36}$ J. Schneider, ${ }^{25}$ F. G. Schröder, ${ }^{30,40}$

L. Schumacher, ${ }^{1}$ S. Sclafani, ${ }^{43}$ D. Seckel, ${ }^{40}$ S. Seunarine, ${ }^{45}$ S. Shefali, ${ }^{1}$ M. Silva, ${ }^{36}$ B. Smithers, ${ }^{4}$ R. Snihur, ${ }^{36}$ J. Soedingrekso, ${ }^{22}$ D. Soldin,${ }^{40}$ M. Song ${ }^{18}$ G. M. Spiczak, ${ }^{45}$ C. Spiering, ${ }^{56, \dagger}$ J. Stachurska,${ }^{56}$ M. Stamatikos,${ }^{20}$ T. Stanev, ${ }^{40}$ R. Stein ${ }^{56}$ J. Stettner, ${ }^{1}$ A. Steuer, ${ }^{37}$ T. Stezelberger, ${ }^{9}$ R. G. Stokstad, ${ }^{9}$ A. Stöß1, ${ }^{15}$ N. L. Strotjohann, ${ }^{56}$ T. Stürwald, ${ }^{1}$

T. Stuttard ${ }^{21}$ G. W. Sullivan, ${ }^{18}$ I. Taboada, ${ }^{6}$ F. Tenholt,${ }^{11}$ S. Ter-Antonyan, ${ }^{7}$ A. Terliuk ${ }^{56}$ S. Tilav,${ }^{40}$ K. Tollefson, ${ }^{23}$

L. Tomankova, ${ }^{11}$ C. Tönnis, ${ }^{50}$ S. Toscano, ${ }^{12}$ D. Tosi, ${ }^{36}$ A. Trettin, ${ }^{56}$ M. Tselengidou, ${ }^{25}$ C. F. Tung, ${ }^{6}$ A. Turcati, ${ }^{26}$

R. Turcotte, ${ }^{30}$ C. F. Turley, ${ }^{53}$ B. Ty, ${ }^{36}$ E. Unger, ${ }^{54}$ M. A. Unland Elorrieta, ${ }^{39}$ M. Usner, ${ }^{56}$ J. Vandenbroucke, ${ }^{36}$

W. Van Driessche, ${ }^{28}$ D. van Eijk ${ }^{36}$ N. van Eijndhoven, ${ }^{13}$ D. Vannerom, ${ }^{14}$ J. van Santen ${ }^{56}$ S. Verpoest,${ }^{28}$ M. Vraeghe, ${ }^{28}$ C. Walck, ${ }^{47}$ A. Wallace, ${ }^{2}$ M. Wallraff, ${ }^{1}$ T. B. Watson, ${ }^{4}$ C. Weaver, ${ }^{24}$ A. Weindl,${ }^{30}$ M. J. Weiss,${ }^{53}$ J. Weldert, ${ }^{37}$ C. Wendt ${ }^{36}$ J. Werthebach, ${ }^{22}$ B. J. Whelan, ${ }^{2}$ N. Whitehorn, ${ }^{33}$ K. Wiebe, ${ }^{37}$ C. H. Wiebusch, ${ }^{1}$ D. R. Williams, ${ }^{51}$ L. Wills, ${ }^{43}$ M. Wolf, ${ }^{26}$ 
T. R. Wood ${ }^{24}$ K. Woschnagg, ${ }^{8}$ G. Wrede, ${ }^{25}$ J. Wulff, ${ }^{11}$ X. W. Xu, ${ }^{7}$ Y. Xu, ${ }^{48}$ J. P. Yanez,${ }^{24}$ G. Yodh, ${ }^{29}$ S. Yoshida, ${ }^{15}$ T. Yuan, ${ }^{36}$ Z. Zhang, ${ }^{48}$ and M. Zöcklein ${ }^{1}$

(IceCube Collaboration)

${ }^{1}$ III. Physikalisches Institut, RWTH Aachen University, D-52056 Aachen, Germany

${ }^{2}$ Department of Physics, University of Adelaide, Adelaide 5005, Australia

${ }^{3}$ Department of Physics and Astronomy, University of Alaska Anchorage, 3211 Providence Drive, Anchorage, Alaska 99508, USA

${ }^{4}$ Department of Physics, University of Texas at Arlington,

502 Yates Street, Science Hall Room 108, Box 19059, Arlington, Texas 76019, USA

${ }^{5}$ CTSPS, Clark-Atlanta University, Atlanta, Georgia 30314, USA

${ }^{6}$ School of Physics and Center for Relativistic Astrophysics, Georgia Institute of Technology, Atlanta, Georgia 30332, USA

${ }^{7}$ Department of Physics, Southern University, Baton Rouge, Lousiana 70813, USA

${ }^{8}$ Department of Physics, University of California, Berkeley, California 94720, USA

${ }^{9}$ Lawrence Berkeley National Laboratory, Berkeley, California 94720, USA

${ }^{10}$ Institut für Physik, Humboldt-Universität zu Berlin, D-12489 Berlin, Germany

${ }^{11}$ Fakultät für Physik \& Astronomie, Ruhr-Universität Bochum, D-44780 Bochum, Germany

${ }^{12}$ Université Libre de Bruxelles, Science Faculty CP230, B-1050 Brussels, Belgium

${ }^{13}$ Vrije Universiteit Brussel (VUB), Dienst ELEM, B-1050 Brussels, Belgium

${ }^{14}$ Department of Physics, Massachusetts Institute of Technology, Cambridge, Massachusetts 02139, USA

${ }^{15}$ Department of Physics and Institute for Global Prominent Research, Chiba University, Chiba 263-8522, Japan

${ }^{16}$ Department of Physics, Loyola University Chicago, Chicago, Illinois 60660, USA

${ }^{17}$ Department of Physics and Astronomy, University of Canterbury, Private Bag 4800, Christchurch, New Zealand

${ }^{18}$ Department of Physics, University of Maryland, College Park, Maryland 20742, USA

${ }^{19}$ Department of Astronomy, Ohio State University, Columbus, Ohio 43210, USA

${ }^{20}$ Department of Physics and Center for Cosmology and Astro-Particle Physics, Ohio State University, Columbus, Ohio 43210, USA

${ }^{21}$ Niels Bohr Institute, University of Copenhagen, DK-2100 Copenhagen, Denmark

${ }^{22}$ Department of Physics, TU Dortmund University, D-44221 Dortmund, Germany

${ }^{23}$ Department of Physics and Astronomy, Michigan State University, East Lansing, Michigan 48824, USA

${ }^{24}$ Department of Physics, University of Alberta, Edmonton, Alberta T6G 2E1, Canada

${ }^{25}$ Erlangen Centre for Astroparticle Physics, Friedrich-Alexander-Universität Erlangen-Nürnberg, D-91058 Erlangen, Germany

${ }^{26}$ Physik-department, Technische Universität München, D-85748 Garching, Germany

${ }^{27}$ Département de physique nucléaire et corpusculaire, Université de Genève, CH-1211 Genève, Switzerland

${ }^{28}$ Department of Physics and Astronomy, University of Gent, B-9000 Gent, Belgium

${ }^{29}$ Department of Physics and Astronomy, University of California, Irvine, California 92697, USA

${ }^{30}$ Karlsruhe Institute of Technology, Institut für Kernphysik, D-76021 Karlsruhe, Germany

${ }^{31}$ Department of Physics and Astronomy, University of Kansas, Lawrence, Kansas 66045, USA

${ }^{32}$ SNOLAB, 1039 Regional Road 24, Creighton Mine 9, Lively, Ontario P3Y 1N2, Canada

${ }^{33}$ Department of Physics and Astronomy, UCLA, Los Angeles, California 90095, USA

${ }^{34}$ Department of Physics, Mercer University, Macon, Georgia 31207-0001, USA

${ }^{35}$ Department of Astronomy, University of Wisconsin, Madison, Wisconsin 53706, USA

${ }^{36}$ Department of Physics and Wisconsin IceCube Particle Astrophysics Center, University of Wisconsin, Madison, Wisconsin 53706, USA

${ }^{37}$ Institute of Physics, University of Mainz, Staudinger Weg 7, D-55099 Mainz, Germany

${ }^{38}$ Department of Physics, Marquette University, Milwaukee, Wisconsin 53201, USA

${ }^{39}$ Institut für Kernphysik, Westfälische Wilhelms-Universität Münster, D-48149 Münster, Germany

${ }^{40}$ Bartol Research Institute and Department of Physics and Astronomy, University of Delaware, Newark, Delaware 19716, USA

${ }^{41}$ Department of Physics, Yale University, New Haven, Connecticut 06520, USA

${ }^{42}$ Department of Physics, University of Oxford, Parks Road, Oxford OXI 3PU, United Kingdom

${ }^{43}$ Department of Physics, Drexel University, 3141 Chestnut Street, Philadelphia, Pennsylvania 19104, USA

${ }^{44}$ Physics Department, South Dakota School of Mines and Technology, Rapid City, South Dakota 57701, USA

${ }^{45}$ Department of Physics, University of Wisconsin, River Falls, Wisconsin 54022, USA

${ }^{46}$ Department of Physics and Astronomy, University of Rochester, Rochester, New York 14627, USA

${ }^{47}$ Oskar Klein Centre and Department of Physics, Stockholm University, SE-10691 Stockholm, Sweden

${ }^{48}$ Department of Physics and Astronomy, Stony Brook University, Stony Brook, New York 11794-3800, USA

${ }^{49}$ Department of Physics, Sungkyunkwan University, Suwon 16419, Korea

${ }^{50}$ Institute of Basic Science, Sungkyunkwan University, Suwon 16419, Korea

${ }^{51}$ Department of Physics and Astronomy, University of Alabama, Tuscaloosa, Alabama 35487, USA 
${ }^{52}$ Department of Astronomy and Astrophysics, Pennsylvania State University, University Park, Pennsylvania 16802, USA

${ }^{53}$ Department of Physics, Pennsylvania State University, University Park, Pennsylvania 16802, USA

${ }^{54}$ Department of Physics and Astronomy, Uppsala University, Box 516, S-75120 Uppsala, Sweden

${ }^{55}$ Department of Physics, University of Wuppertal, D-42119 Wuppertal, Germany

${ }^{56}$ DESY, D-15738 Zeuthen, Germany

${ }^{57}$ Institute for Cosmic Ray Research, the University of Tokyo,

5-1-5 Kashiwa-no-ha, Kashiwa, Chiba 277-8582, Japan

(Received 8 June 2020; accepted 31 August 2020; published 30 September 2020)

The results of a $3+1$ sterile neutrino search using eight years of data from the IceCube Neutrino Observatory are presented. A total of 305735 muon neutrino events are analyzed in reconstructed energyzenith space to test for signatures of a matter-enhanced oscillation that would occur given a sterile neutrino state with a mass-squared differences between 0.01 and $100 \mathrm{eV}^{2}$. The best-fit point is found to be at $\sin ^{2}\left(2 \theta_{24}\right)=0.10$ and $\Delta m_{41}^{2}=4.5 \mathrm{eV}^{2}$, which is consistent with the no sterile neutrino hypothesis with a $p$ value of $8.0 \%$.

DOI: 10.1103/PhysRevLett.125.141801

Introduction.-The three-flavor massive neutrino oscillation formalism has been well-established experimentally [1-4]. The standard paradigm has also been challenged, by several experiments exhibiting anomalous $\nu_{e}\left(\bar{\nu}_{e}\right)$ appearance in $\nu_{\mu}\left(\bar{\nu}_{\mu}\right)$ beams $[5,6]$. These anomalies can be interpreted as evidence for subleading oscillations of $\nu_{\mu} \rightarrow \nu_{e}$ or $\bar{\nu}_{\mu} \rightarrow \bar{\nu}_{e}$ caused by additional neutrinos with large mass-squared differences in the range of $\Delta m^{2} \sim$ $0.1-10 \mathrm{eV}^{2}$ [7-11]. On the other hand, measurements of the $Z$-boson decay width to invisible final states demonstrate that only three light neutrinos participate in weak interactions [12], so any additional neutrino flavor states must be nonweakly interacting, or "sterile." The simplest such model is referred to as a " $3+1$ " model, where in addition to the three known mass states, a fourth heavier one is added.

The relationship between the flavor and mass states is described by a unitary matrix, $U_{\mathrm{PNMS}}$, which in the threeneutrino model can be parameterized in terms of three mixing angles and one oscillation-accessible $C P$-violating phase. Adding a sterile state expands the mixing matrix to four dimensions, in which the added degrees of freedom can be parameterized by introducing three new rotations with angles $\theta_{14}, \theta_{24}$, and $\theta_{34}$, and two new oscillationaccessible $C P$-violating phases, $\delta_{14}$ and $\delta_{24}$. The oscillation phenomenology of the $3+1$ model adds both shorter baseline vacuumlike oscillations, and also novel oscillation effects in the presence of matter [13-17]. For eV-scale sterile neutrino states, for example, a matter-enhanced resonance [18-23] would result in the near complete disappearance of $\mathrm{TeV}$-scale muon antineutrinos passing

Published by the American Physical Society under the terms of the Creative Commons Attribution 4.0 International license. Further distribution of this work must maintain attribution to the author(s) and the published article's title, journal citation, and DOI. Funded by SCOAP ${ }^{3}$. through the Earth's core, as shown in Fig. 1. By measuring and characterizing the flux of atmospheric neutrinos in the $\mathrm{GeV}$ to $\mathrm{PeV}$ energy range, the IceCube Neutrino Observatory is uniquely positioned to search for such matter-enhanced oscillations, a smoking-gun signature of $\mathrm{eV}$-scale sterile neutrinos.

Testing the $3+1$ model as an explanation of shortbaseline anomalies and constraining its free parameters requires measurements in multiple oscillation channels,

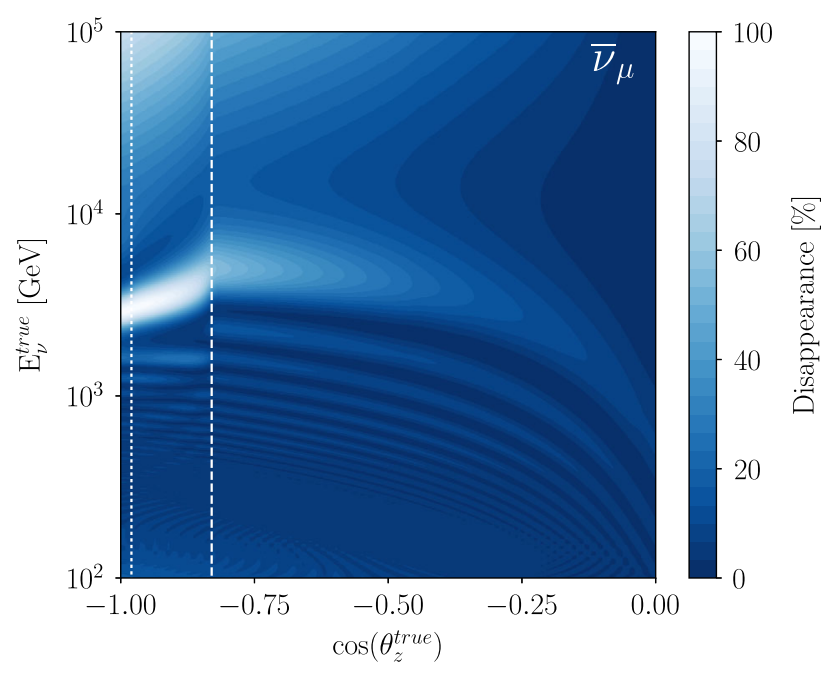

FIG. 1. Muon-antineutrino oscillogram. Atmospheric $\bar{\nu}_{\mu}$ disappearance probability vs true energy and cosine zenith at the globally preferred sterile neutrino hypothesis of Ref. [11] $\left[\Delta m_{41}^{2}=1.3 \mathrm{eV}^{2}, \sin ^{2}\left(2 \theta_{24}\right)=0.07, \sin ^{2}\left(2 \theta_{34}\right)=0.0\right]$. Effects include a matter-enhanced resonance at $\mathrm{TeV}$ energies, neutrino absorption at high energy and small zenith, and vacuumlike oscillation at low energies. The matter-enhanced resonance appears only in the antineutrino flux for the case of small angles and $\Delta m_{41}^{2}>0$. Vertical white lines indicate transitions between inner to outer core $\left[\cos \left(\theta_{\nu}^{\text {true }}\right)=-0.98\right]$ and outer core to mantle $\left[\cos \left(\theta_{z}^{\text {true }}\right)=-0.83\right]$. 
including $\nu_{\mu} \rightarrow \nu_{\mu}$ [24-32], $\nu_{e} \rightarrow \nu_{e}$ [33-41], and $\nu_{\mu} \rightarrow \nu_{e}$ $[5,6,42-44]$. Fits to global data $[9,11,45]$ find a strong preference for models with sterile neutrinos over the standard three-neutrino paradigm. However, even at the most preferred values of $\Delta m^{2} \sim 1 \mathrm{eV}^{2}$, the mixing angles required to viably explain anomalies in the $\nu_{\mu} \rightarrow \nu_{e}$ and $\bar{\nu}_{\mu} \rightarrow \bar{\nu}_{e}$ channels are in strong tension with measurements of $\nu_{\mu}$ and $\bar{\nu}_{\mu}$ disappearance $[11,45]$. These are also in tension with cosmological observations [46-52], though a number of possible solutions have been proposed [53-63]. Evidence for oscillation effects beyond the three-neutrino paradigm in $\bar{\nu}_{\mu}$ disappearance are yet to be observed [45]. One of these nonobservations was made by IceCube, using a sample of 20145 atmospheric $\nu_{\mu}$ and $\bar{\nu}_{\mu}$ events collected over one year of detector livetime $[29,64]$.

This Letter updates IceCube's high-energy sterile neutrino search using an eight-year dataset and improved event selection. The sample includes 305735 well-reconstructed charged-current $\nu_{\mu}$ and $\bar{\nu}_{\mu}$ events collected from May 13, 2011, to May 19, 2019. Events are binned uniformly in $\log \left(E_{\text {reco }}^{\mu}\right)$ spanning $E_{\text {reco }}^{\mu} \in[500 \mathrm{GeV}, 9976 \mathrm{GeV}]$ in 13 bins and uniformly in $\cos \theta_{z}^{\text {reco }}$ spanning the up-going region from -1.0 to 0.0 in 20 bins. The event counts in each bin are used as inputs to a likelihood-based analysis to test for evidence of $\mathrm{eV}$-scale sterile neutrinos.

The increased sample size of this analysis with respect to Ref. [29] has been accompanied by a commensurate improvement in the precision of treatments of systematic uncertainties and statistical methods. This Letter summarizes these advances and presents the main results of this search. A companion paper, Ref. [65], contains a more detailed exposition of the technical aspects of the analysis, as well as alternate interpretations of the data in a wider space of sterile neutrino parameters.

IceCube up-going track sample.-The IceCube Neutrino Observatory is a cubic-kilometer neutrino detector buried in the Antarctic glacier [66]. It is comprised of photomultiplier tubes enclosed in glass pressure housings called "digital optical modules" (DOMs) [67]. These are arranged in vertical strings on a hexagonal lattice. The main array consists of 78 strings spaced $125 \mathrm{~m}$ apart, each supporting 60 downward-facing DOMs with a $17 \mathrm{~m}$ vertical spacing. A denser array called DeepCore [68] instruments the clearest part of the ice within the main array. The eight strings of DeepCore are arranged with lateral spacing between 42 and $72 \mathrm{~m}$ and vertical DOM separation of $7 \mathrm{~m}$. This analysis uses the complete set of IceCube DOMs in both the main array and DeepCore.

The majority of IceCube events are produced by highenergy muons and neutrinos from cosmic-ray air showers. Down-going $\left(\cos \theta_{z}^{\text {true }}>0\right)$ atmospheric muons (and antimuons) can penetrate the $1450 \mathrm{~m}$ vertical overburden of the detector, triggering at a rate of $\sim 3 \mathrm{kHz}$ [69]. These events dominate the southern-hemisphere through-going sample. Up-going atmospheric muons, on the other hand, are effectively removed by the large overburden provided by the Earth. Thus, muons originating from the northern hemisphere are dominated by those produced in chargedcurrent neutrino interactions. A charged-current $\nu_{\mu}$ interaction will produce a forward secondary muon with an energy typically between $50 \%$ and $80 \%$ of that of the parent $\nu_{\mu}$ [70]. The muon travels through the ice emitting Cherenkov radiation. While photons travel tens to hundreds of meters before being absorbed by the impurities in the ice [71-73], muons with $\mathrm{TeV}$ energies are able to penetrate multiple kilometers of ice before falling below the Cherenkov threshold $[74,75]$. This produces an extended tracklike signature. These events originate either inside of the detector or from a target volume extending meters to kilometers outside the array, depending on energy [74,76].

Events used in this analysis first pass a filter that selects muonlike events for satellite transmission to the north, and are then subject to further data-reduction techniques to reject low-energy and poorly reconstructed tracks. Only data periods with 86 active IceCube strings and greater than 5000 active DOMs in the detector are considered. A highlevel event selection is applied, leveraging morphology, measures of track reconstruction quality, and the expected transmission of signal events through the zenith-dependent overburden, explained in detail in Ref. [65] and based on Ref. [77]. The reconstructed energy and direction of each event is calculated according to the time and geometry of light detected throughout the array $[78,79]$. The angular resolution $\sigma_{\cos \theta_{z}}$ varies between 0.005 and 0.015 and energy resolution of $\sigma_{\log _{10} E_{\mu}} \sim 0.5$, as in the previous version of this analysis [29]. The energy distribution of selected events is shown in Fig. 2.

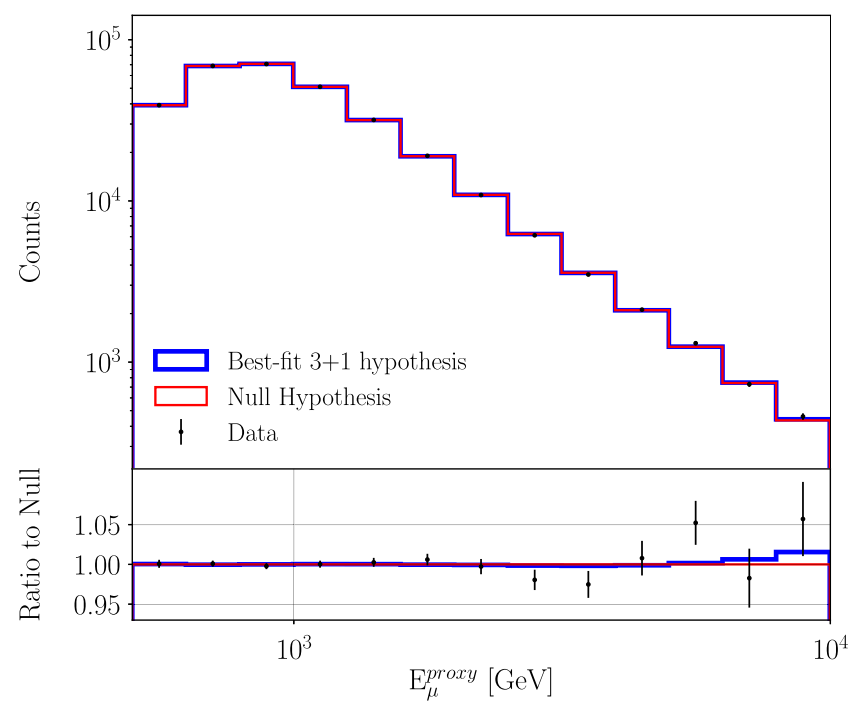

FIG. 2. Reconstructed muon energy. Data points are shown as black markers with error bars that represent the statistical error. The solid blue and red lines show the best-fit sterile neutrino hypothesis and the null (no sterile neutrino) hypothesis, respectively, with nuisance parameters set to their best-fit values in each case. 
Cosmic-ray muon background contamination is assessed using CORSIKA [80], with primary cosmic-ray energies ranging from 600 to $10^{11} \mathrm{GeV}$. Approximately $10 \%$ of the dataset of neutrino events are predicted to contain a coincident cosmic-ray muon within the readout frame. The $\nu_{\mu}$ and cosmic-ray muon tracks are separated into sub-events using an event splitter, and each subevent is treated independently in the event selection. After splitting and event selection, the sample is predicted to be $>99.9 \%$ pure in $\nu_{\mu} / \bar{\nu}_{\mu}$ induced events [65].

Sterile meutrino snalysis.-In this analysis, we consider a sterile neutrino model parametrized by one mass-squared difference, $\Delta m_{41}^{2}$, and one mixing angle, $\sin ^{2}\left(\theta_{24}\right)$. For each hypothesis point on a grid of $\Delta m_{41}^{2}$ from $10^{-2}$ to $10^{2} \mathrm{eV}^{2}$ and $\sin ^{2}\left(2 \theta_{24}\right)$ from $10^{-3}$ to 1 , the neutrino flux incident on the detector is calculated using the four-flavor formalism.

The neutrino flux includes contributions from both atmospheric and astrophysical neutrinos. The conventional atmospheric $\nu_{\mu}$ and $\bar{\nu}_{\mu}$ flux is produced by the decay of pions and kaons and is calculated using the MCEq cascade equation solver [81,82]. The hadronic interactions are modeled with SIBYLL2.3c [83]. The primary cosmic-ray spectrum is a three-population model $[84,85]$, in which each population contains five groups of nuclei. The zenithdependent seasonal atmospheric density profile, through which the cascade develops, is determined using data from the atmospheric infrared sounder (AIRS) satellite [86]. The prompt $\nu_{\mu}$ component from the decay of charmed mesons is implemented as in Ref. [87]. The astrophysical neutrino flux is assumed to have equal parts of each neutrino flavor and to be symmetric in neutrinos and antineutrinos [88-90]; be isotropically distributed; and have a single power-law energy spectrum consistent with previous IceCube measurements [91]. These fluxes are subject to a suite of systematic uncertainties, summarized in the following section.

For each sterile neutrino hypothesis, the atmospheric and astrophysical neutrino fluxes are propagated through the Earth using the nuSQuIDS neutrino evolution code $[92,93]$. This accounts for both coherent and noncoherent interactions [94]: namely charged-current, neutral-current, and Glashow resonance interactions [95], as well as tauneutrino regeneration [96]. We use the CSMS [97] neutrino-nucleon cross section to describe both interactions during neutrino propagation and near the detector. This requires as an input the Earth density profile, which we parametrize via the spherically symmetric PREM model [98]. Using the above, we obtain a prediction for the up-going $\nu_{\mu}$ flux at the detector for each physics parameter point. These fluxes are used to weight detector Monte Carlo (MC) event sets, with effective livetime $\geq 50 \times$ the sample size.

We account for systematic uncertainties by means of nuisance parameters, which reweight the MC event sets by applying continuous parametrizations of the effects discussed in the following section. We then compare the data to expectation using a modified version of the Poisson likelihood to account for MC statistical uncertainty [99]. For our frequentist analysis, the likelihood is profiled over the eighteen nuisance parameters to construct a test statistic. Frequentist contours are constructed using Wilks's theorem [100], validated at an array of parameter points using MC ensembles and the Feldman-Cousins [101] procedure. A Bayesian hypothesis test is also performed, by means of comparing the model evidences [102] with respect to the no sterile neutrino hypothesis. The model evidences, as a function of sterile neutrino parameters, are computed by integrating the likelihood over the nuisance parameters using MultiNest [103]. These two statistical approaches are complementary: the Bayesian approach conveys the likelihood of the model given observed data and prior knowledge, whereas the frequentist approach yields intervals that are likely to contain the true model parameters for repeated experiments, enabling direct comparison with previous publications.

Systematic uncertainties.-Dominant sources of uncertainty derive from the shape and normalization of astrophysical and atmospheric neutrino fluxes; the bulk properties of the South Pole ice; the local response of the IceCube DOMs; and neutrino interaction cross sections. Other uncertainties, such as the Earth density profile, neutrino interactions in the rock and ice transition region, prompt neutrino flux, and $\nu_{\mu} / \bar{\nu}_{\mu}$ astrophysical ratio were investigated but established as negligible relative to statistical uncertainty.

Atmospheric neutrino flux:In the relevant energy range the spectrum of cosmic-ray primaries follows approximately an $E^{-2.65}$ energy $(E)$ dependence. To account for the uncertainty in the cosmic-ray spectral index, we apply a spectral shift $\Delta \gamma$ with an uncertainty of 0.03 pivoting at $2.2 \mathrm{TeV}$ [104-107]. The meson production uncertainty in the interaction between the primary cosmic ray and air and in subsequent hadronic interactions is described through the Barr et al. scheme [108]. In this scheme, the uncertainty in the differential cross section for meson production is quantified in regions of primary proton energy $E_{p}$ and meson fractional momenta $x_{\mathrm{lab}}$. The charged-kaon production yield carries the leading uncertainty. We parametrize its production over three kinematic regions: $x_{\text {lab }}<0.1$ and $E_{p}>30 \mathrm{GeV} ; x_{\mathrm{lab}} \geq 0.1$ and $30 \mathrm{GeV}<E_{p}<500 \mathrm{GeV}$; and $x_{\mathrm{lab}}>0.1$ and $E_{p} \geq 500 \mathrm{GeV}$. We include two collider-constrained nuisance parameters for each region, one for particles and one for antiparticles, which rescale the production cross section. The highest-energy uncertainties are obtained through extrapolation, and both the scale and energy dependence have ascribed uncertainties. Kaon energy losses by interaction with oxygen and nitrogen nuclei are accounted for via the total kaon-nucleus cross sectional uncertainty [109]. The charged-pion production 
and interaction uncertainties were studied and found negligible. The atmospheric density profile is inferred from the zenith-dependent vertical temperature profile measured by the AIRS satellite. To incorporate its uncertainty, showers are recomputed through randomly perturbed density models within the statistical and systematic uncertainties reported in the AIRS measurements. Finally, the total conventional atmospheric $\nu_{\mu}$ flux carries an additional $40 \%$ normalization uncertainty following Ref. [82].

Astrophysical neutrino flux:The central astrophysical model is a single power law with an equal normalization for all neutrino and antineutrino flavors at $100 \mathrm{TeV}$ of $0.787 \times 10^{-18} \mathrm{GeV}^{-1} \mathrm{sr}^{-1} \mathrm{~s}^{-1} \mathrm{~cm}^{-2}$ and a spectral index of 2.5. The Gaussian priors on the normalization and spectral index are correlated and selected to accommodate all IceCube astrophysical neutrino flux measurements to date [91,110-114], with allowed spectral indices of $\gamma_{\text {astro }} \sim$ $2.2-2.8$ at $68 \%$ confidence level (C.L.). This represents a significant contribution to the total flux in the top two energy bins, depending strongly on the value of $\gamma_{\text {astro }}$.

Bulk ice model:The uncertainty associated with the measured scattering and absorption of the undisturbed glacial ice is implemented as described in Ref. [115]. This treatment expresses the depth dependence of the ice optical properties using a Fourier decomposition. The covariance of the Fourier mode coefficients are determined using LED flasher calibration data [73]. Only the six lowest modes contribute a sizeable shape difference in the reconstructed event distributions. The effect of these modes is parametrized using two empirical energy-dependent basis functions. The two associated amplitudes are incorporated as nuisance parameters with a correlated bivariate Gaussian prior.

DOM response and local ice effects:The ice in the immediate vicinity of the DOMs has optical properties distinct from the bulk ice between strings [116], caused by bubble formation during the refreezing process after their deployment. This introduces uncertainties via two effects. First, the global photon detection efficiency is impacted [117]. This is modeled by an efficiency correction with an effectively flat prior, ultimately constrained with a tight posterior through its effect on the overall energy scale. Second, the bubble column influences the angular dependence of photon detection. This is encoded in two parameters tuned to detailed optical simulations of bubble scattering near the DOM [118], with only one having a substantial impact.

Neutrino cross section:The neutrino-nucleon cross section enters the analysis in two ways, influencing (1) the absorption during the neutrino propagation through the Earth [70,119] and (2) the rates and inelasticities of interactions near the detector $[70,97,120]$. The latter source of uncertainty was previously investigated in Refs. [121,122] and found to be negligible. The former is found to be non-negligible and is taken into account by separately parametrizing the change in neutrino absorption when the $\nu_{\mu}$ and $\bar{\nu}_{\mu}$ cross sections are scaled. The priors on these parameters are fixed at the largest uncertainties in our energy range from Ref. [97], which are $3 \%$ for $\nu_{\mu}$ and $7 \%$ for $\bar{\nu}_{\mu}$.

Results.-The frequentist analysis best-fit point is $\Delta m_{41}^{2}=4.5 \mathrm{eV}^{2}$ and $\sin ^{2}\left(2 \theta_{24}\right)=0.10$. At this point, the largest nuisance parameter pull was observed in the cosmic-ray spectral index, which shifted the cosmic-ray spectrum by $0.068(2.3 \sigma)$; the other nuisance parameter best-fit values are within one sigma of their respective central values and can be found in the accompanying Ref. [65]. Figure 3 shows the signal shape at the best-fit point, given the best-fit nuisance parameters, as well as the pull between data and no sterile neutrino hypothesis, evaluated at those same nuisance parameters. Figure 4 shows the $90 \%$ and $99 \%$ C.L. contours calculated according to Wilks's theorem with two degrees of freedom. Sensitivity envelopes, illustrating symmetrically counted ensembles of $68 \%$ and $95 \%$ nonclosed contours derived from 2000 pseudoexperiments, are shown overlaid for the
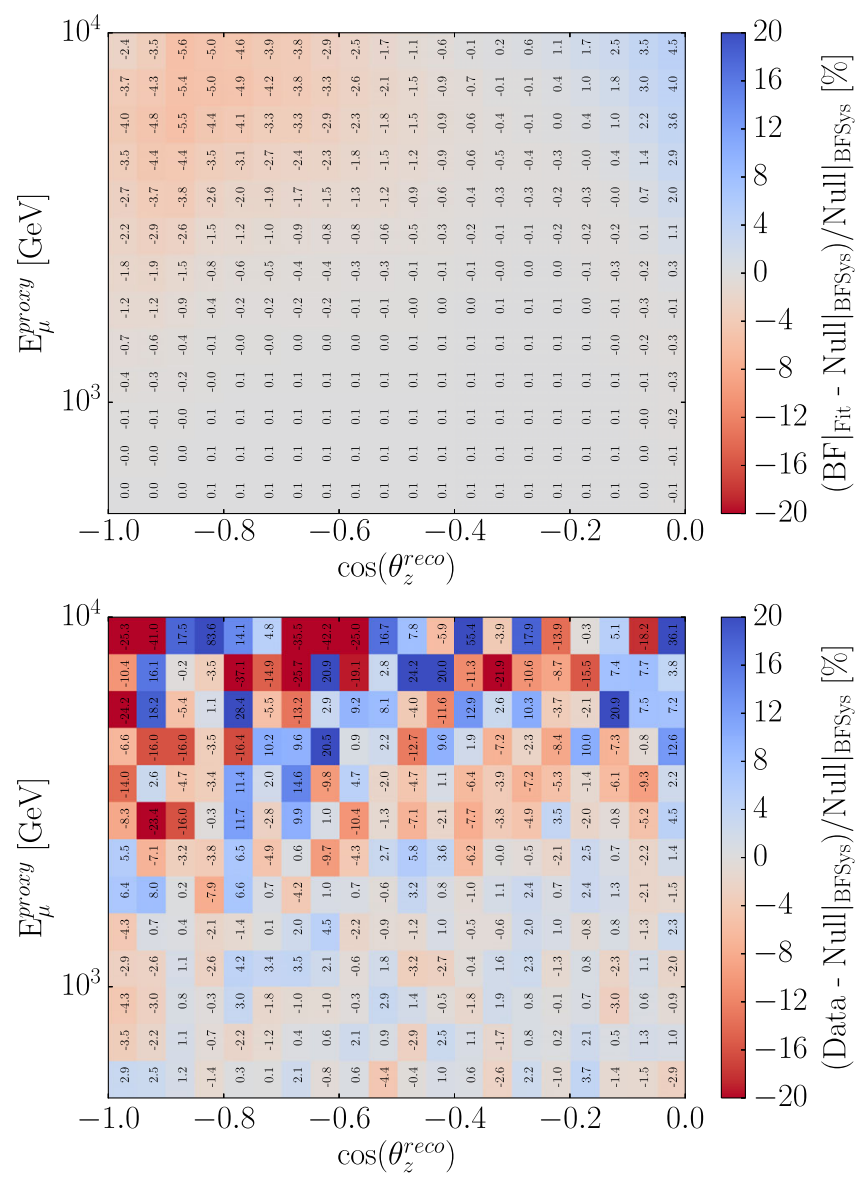

FIG. 3. Best-fit signal shapes compared to data. Top: the signal shape at the best-fit point compared to the null hypothesis with the same nuisance parameters. Bottom: data compared to the null hypothesis with the nuisance parameters held at the same values. 


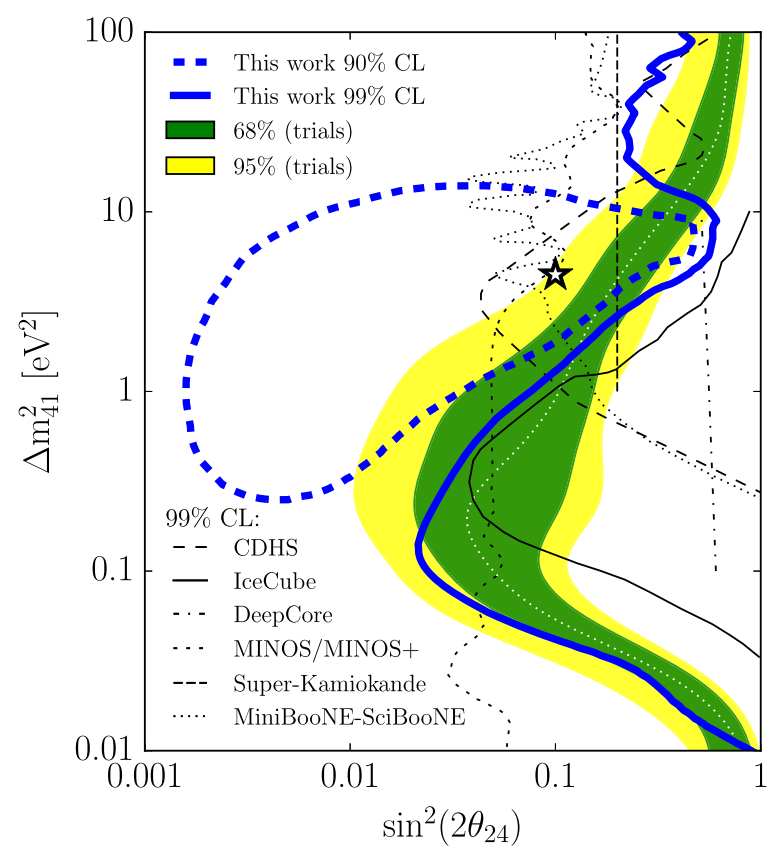

FIG. 4. Frequentist analysis result. The $90 \%$ and $99 \%$ C.L. contours, assuming Wilks's theorem, shown as dashed and solid bold blue lines, respectively. The green and yellow band shows the region where $68 \%$ and $95 \%$ of the pseudoexperiment 99\% C.L. observations lie; the dashed white line corresponds to the median. Other muon-neutrino disappearance measurements at $99 \%$ C.L. are shown in black [25-30,123,124]; where results were not available at 99\% C.L., methods of Ref. [11] were applied using public data releases. Finally, the star marks the analysis best-fit point location.

99\% contour. The IceCube $90 \%$ C.L. preferred region is consistent with constraints from previous $\nu_{\mu}$ disappearance experiments, and the $99 \%$ contour is stronger than other exclusion limits at values of $\Delta m^{2}$ up to $1 \mathrm{eV}^{2}$.

Figure 5 shows the corresponding Bayesian result, where the pointwise Bayes factor is calculated relative to the no sterile neutrino hypothesis. The best-model location is at $\Delta m^{2} \sim 4.5 \mathrm{eV}^{2}$ and $\sin ^{2}\left(2 \theta_{24}\right) \sim 0.9$ and is strongly preferred, by a factor of 10.7 , to the no sterile neutrino hypothesis. Contours are drawn in logarithmic Bayes factor steps of 0.5, quantifying strength of evidence [125].

The best-fit point and inferred confidence regions are found to be robust under the removal of any one of the eight years of data, showing only minor changes in the contour position. This is also the case for removal of any of the following group of uncertainties: neutrino cross sections, detector effects, atmospheric flux, and astrophysical flux. Details can be found in Refs. [65,126]. Furthermore, a similar best-fit point is obtained when fitting any one year of data independently, suggesting a small effect of physical or systematic rather than statistical origin.

The difference in likelihood to the null hypothesis is 4.94 , corresponding to a $p$ value of $8 \%$ against the null hypothesis. The location of this point was found to be

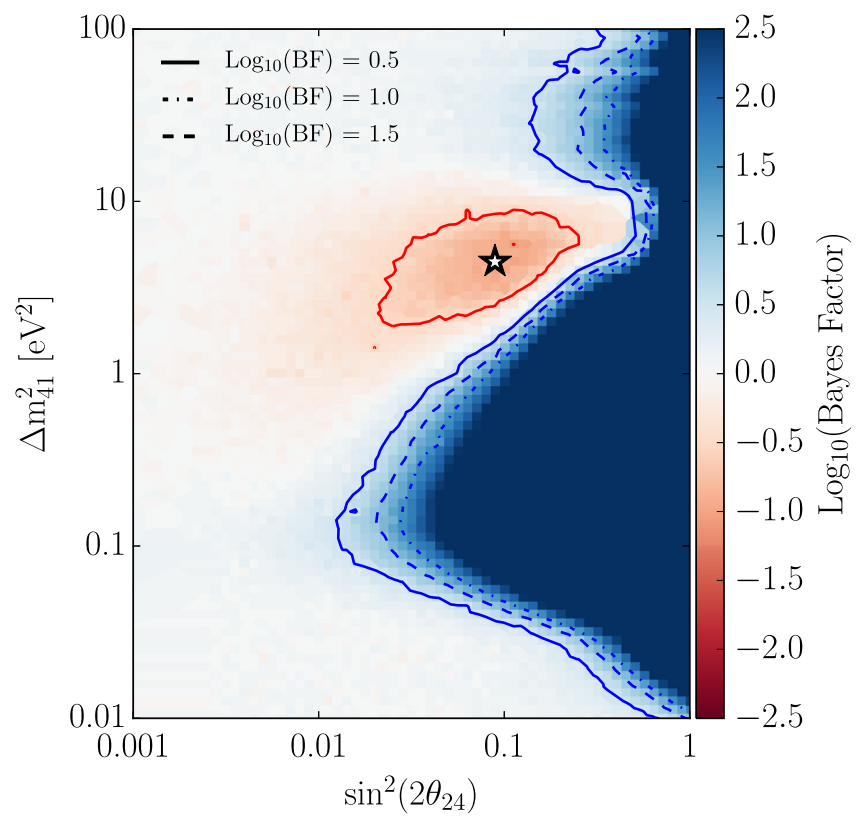

FIG. 5. Bayesian analysis result. The logarithm of the Bayes factor [125] relative to the null hypothesis (color scale). Red indicates hypotheses preferred over the null hypothesis, while the blue indicates the null is preferred. Solid lines delineate likelihood ratios of 1 in 10 for a priori equally likely hypotheses. The best-model location is shown at the white star with a $\log _{10}$ (Bayes factor) minimum of -1.03 .

compatible with expectations based on simulated no sterile neutrino pseudoexperiments, which by definition produce closed contours at $90 \%$ C.L. in $10 \%$ of trials.

In summary, we have studied 305735 up-going atmospheric and astrophysical muon neutrinos to search for evidence of eV-sterile neutrino signatures. The best-fit point is consistent with the no sterile neutrino hypothesis at a $p$ value of $8 \%$. Because of its unique statistical strength this result is expected to have a substantial impact on the global sterile neutrino landscape.

The IceCube collaboration acknowledges the significant contributions to this manuscript from the Massachusetts Institute of Technology and University of Texas at Arlington groups.

We acknowledge the support from the following agencies: USA-U.S. National Science FoundationOffice of Polar Programs, U.S. National Science Foundation-Physics Division, Wisconsin Alumni Research Foundation, Center for High Throughput Computing (CHTC) at the University of WisconsinMadison, Open Science Grid (OSG), Extreme Science and Engineering Discovery Environment (XSEDE), U.S. Department of Energy-National Energy Research Scientific Computing Center, Particle astrophysics research computing center at the University of Maryland, Institute for Cyber-Enabled Research at Michigan State University, and Astroparticle physics computational facility at Marquette 
University; Belgium-Funds for Scientific Research (FRS-FNRS and FWO), FWO Odysseus and Big Science programmes, and Belgian Federal Science Policy Office (Belspo); Germany-Bundesministerium für Bildung und Forschung (BMBF), Deutsche Forschungsgemeinschaft (DFG), Helmholtz Alliance for Astroparticle Physics (HAP), Initiative and Networking Fund of the Helmholtz Association, Deutsches Elektronen Synchrotron (DESY), and High Performance Computing cluster of the RWTH Aachen; Sweden-Swedish Research Council, Swedish Polar Research Secretariat, Swedish National Infrastructure for Computing (SNIC), and Knut and Alice Wallenberg Foundation; Australia-Australian Research Council; Canada-Natural Sciences and Engineering Research Council of Canada, Calcul Québec, Compute Ontario, Canada Foundation for Innovation, WestGrid, and Compute Canada; DenmarkVillum Fonden, Danish National Research Foundation (DNRF), Carlsberg Foundation; New Zealand-Marsden Fund; Japan-Japan Society for Promotion of Science (JSPS) and Institute for Global Prominent Research (IGPR) of Chiba University; Korea-National Research Foundation of Korea (NRF); Switzerland-Swiss National Science Foundation (SNSF); United KingdomDepartment of Physics, University of Oxford.

*Also at Università di Padova, I-35131 Padova, Italy ${ }^{\dagger}$ Also at National Research Nuclear University, Moscow Engineering Physics Institute (MEPhI), Moscow 115409, Russia.

Earthquake Research Institute, University of Tokyo, Bunkyo, Tokyo 113-0032, Japan.

[1] M. Tanabashi et al. (Particle Data Group), Review of particle physics, Phys. Rev. D 98, 030001 (2018).

[2] I. Esteban, M. C. Gonzalez-Garcia, A. HernándezCabezudo, M. Maltoni, and T. Schwetz, Global analysis of three-flavour neutrino oscillations: Synergies and tensions in the determination of $\theta_{23}, \delta_{C P}$, and the mass ordering, J. High Energy Phys. 01 (2019) 106.

[3] P. F. de Salas, D. V. Forero, C. A. Ternes, M. Tortola, and J. W. F. Valle, Status of neutrino oscillations 2018: $3 \sigma$ hint for normal mass ordering and improved $C P$ sensitivity, Phys. Lett. B 782, 633 (2018).

[4] F. Capozzi, E. Lisi, A. Marrone, D. Montanino, and A. Palazzo, Neutrino masses and mixings: Status of known and unknown $3 \nu$ parameters, Nucl. Phys. B908, 218 (2016).

[5] C. Athanassopoulos, L. B. Auerbach, R. L. Burman, D. O. Caldwell, E. D. Church et al. (LSND Collaboration), Evidence for $\nu_{\mu} \rightarrow \nu_{e}$ Neutrino Oscillations from LSND, Phys. Rev. Lett. 81, 1774 (1998).

[6] A. A. Aguilar-Arevalo, B. C. Brown, L. Bugel, G. Cheng, J. M. Conrad et al. (MiniBooNE Collaboration), Significant Excess of ElectronLike Events in the MiniBooNE Short-Baseline Neutrino Experiment, Phys. Rev. Lett. 121, 221801 (2018).
[7] M. Cirelli, G. Marandella, A. Strumia, and F. Vissani, Probing oscillations into sterile neutrinos with cosmology, astrophysics and experiments, Nucl. Phys. B708, 215 (2005).

[8] K. Abazajian et al., Light sterile neutrinos: A white paper, arXiv:1204.5379.

[9] S. Gariazzo, C. Giunti, M. Laveder, and Y. F. Li, Updated global $3+1$ analysis of short-baseline neutrino oscillations, J. High Energy Phys. 06 (2017) 135.

[10] M. Dentler, A. Hernández-Cabezudo, J. Kopp, M. Maltoni, and T. Schwetz, Sterile neutrinos or flux uncertainties?Status of the reactor anti-neutrino anomaly, J. High Energy Phys. 11 (2017) 099.

[11] A. Diaz, C. Argüelles, G. Collin, J. Conrad, and M. Shaevitz, Where are we with light sterile neutrinos? Phys. Rep. (2020), https://doi.org/10.1016/j.physrep.2020.08.005.

[12] S. Schael et al. (ALEPH, DELPHI, L3, OPAL, SLD Collaborations, LEP Electroweak Working Group, SLD Electroweak Group, SLD Heavy Flavour Group), Precision electroweak measurements on the $Z$ resonance, Phys. Rep. 427, 257 (2006).

[13] E. K. Akhmedov, Neutrino oscillations in inhomogeneous matter, Yad. Fiz. 47, 475 (1988) [Sov. J. Nucl. Phys. 47, 301 (1988) (in Russian)].

[14] P. I. Krastev and A. Yu. Smirnov, Parametric effects in neutrino oscillations, Phys. Lett. B 226, 341 (1989).

[15] M. Chizhov, M. Maris, and S. T. Petcov, On the oscillation length resonance in the transitions of solar and atmospheric neutrinos crossing the earth core, arXiv:hep-ph/9810501.

[16] M. V. Chizhov and S. T. Petcov, New Conditions for a Total Neutrino Conversion in a Medium, Phys. Rev. Lett. 83, 1096 (1999).

[17] E. K. Akhmedov and A. Yu. Smirnov, Comment on 'New Conditions for a Total Neutrino Conversion in a Medium', Phys. Rev. Lett. 85, 3978 (2000).

[18] H. Nunokawa, O. L. G. Peres, and R. Zukanovich, Funchal, probing the LSND mass scale and four neutrino scenarios with a neutrino telescope, Phys. Lett. B 562, 279 (2003).

[19] S. Choubey, Signature of sterile species in atmospheric neutrino data at neutrino telescopes, J. High Energy Phys. 12 (2007) 014.

[20] V. Barger, Y. Gao, and D. Marfatia, Is there evidence for sterile neutrinos in IceCube data? Phys. Rev. D 85, 011302 (R) (2012).

[21] A. Esmaili, F. Halzen, and O. L. G. Peres, Constraining sterile neutrinos with AMANDA and IceCube atmospheric neutrino data, J. Cosmol. Astropart. Phys. 11 (2012) 041.

[22] A. Esmaili and A. Yu. Smirnov, Restricting the LSND and MiniBooNE sterile neutrinos with the IceCube atmospheric neutrino data, J. High Energy Phys. 12 (2013) 014.

[23] M. Lindner, W. Rodejohann, and X.-J. Xu, Sterile neutrinos in the light of IceCube, J. High Energy Phys. 01 (2016) 124.

[24] F. Dydak et al., A search for muon-neutrino oscillations in the $\Delta \mathrm{m}^{2}$ range $0.3 \mathrm{eV}^{2}$ to $90 \mathrm{eV}^{2}$, Phys. Lett. 134B, 281 (1984). 
[25] I. E. Stockdale, A. Bodek, F. Borcherding, N. Giokaris, K. Lang et al., Limits on Muon Neutrino Oscillations in the Mass Range $55 \mathrm{ev}^{2}<\Delta m^{2}<800 \mathrm{eV}^{2}$, Phys. Rev. Lett. 52, 1384 (1984).

[26] K. B. M. Mahn et al. (SciBooNE and MiniBooNE Collaborations), Dual baseline search for muon neutrino disappearance at $0.5 \mathrm{eV}^{2}<\Delta \mathrm{m}^{2}<40 \mathrm{eV}^{2}$, Phys. Rev. D 85, 032007 (2012).

[27] K. Abe et al. (Super-Kamiokande Collaboration), Limits on sterile neutrino mixing using atmospheric neutrinos in Super-Kamiokande, Phys. Rev. D 91, 052019 (2015).

[28] P. Adamson et al. (MINOS Collaboration), Search for Sterile Neutrinos Mixing with Muon Neutrinos in MINOS, Phys. Rev. Lett. 117, 151803 (2016).

[29] M. G. Aartsen et al. (IceCube Collaboration), Searches for Sterile Neutrinos with the IceCube Detector, Phys. Rev. Lett. 117, 071801 (2016).

[30] M. G. Aartsen et al. (IceCube Collaboration), Search for sterile neutrino mixing using three years of IceCube DeepCore data, Phys. Rev. D 95, 112002 (2017).

[31] P. Adamson et al. (MINOS+ Collaboration), Search for Sterile Neutrinos in MINOS and MINOS+ Using a TwoDetector Fit, Phys. Rev. Lett. 122, 091803 (2019).

[32] A. Albert et al. (ANTARES Collaboration), Measuring the atmospheric neutrino oscillation parameters and constraining the $3+1$ neutrino model with ten years of ANTARES data, J. High Energy Phys. 06 (2019) 113.

[33] Y. Declais et al., Search for neutrino oscillations at 15meters, 40-meters, and 95-meters from a nuclear power reactor at Bugey, Nucl. Phys. B434, 503 (1995).

[34] J. N. Abdurashitov, V. N. Gavrin, V. V. Gorbachev, P. P. Gurkina, T. V. Ibragimova et al. (SAGE Collaboration), Measurement of the solar neutrino capture rate with gallium metal. III: Results for the 2002-2007 data-taking period, Phys. Rev. C 80, 015807 (2009).

[35] F. Kaether, W. Hampel, G. Heusser, J. Kiko, and T. Kirsten, Reanalysis of the GALLEX solar neutrino flux and source experiments, Phys. Lett. B 685, 47 (2010).

[36] J. M. Conrad and M. H. Shaevitz, Limits on electron neutrino disappearance from the KARMEN and LSND $\nu_{e}$-Carbon cross section data, Phys. Rev. D 85, 013017 (2012).

[37] Y. J. Ko, B. R. Kim, J. Y. Kim, B. Y. Han, C. H. Jang et al. (NEOS Collaboration), Sterile Neutrino Search at the NEOS Experiment, Phys. Rev. Lett. 118, 121802 (2017).

[38] F. P. An et al. (Daya Bay Collaboration), Improved Search for a Light Sterile Neutrino with the Full Configuration of the Daya Bay Experiment, Phys. Rev. Lett. 117, 151802 (2016).

[39] I. Alekseev et al. (DANSS Collaboration), Search for sterile neutrinos at the DANSS experiment, Phys. Lett. B 787, 56 (2018).

[40] J. Ashenfelter (PROSPECT Collaboration), First Search for Short-Baseline Neutrino Oscillations at HFIR with PROSPECT, Phys. Rev. Lett. 121, 251802 (2018).

[41] H. Almazán, P. del Amo Sanchez, L. Bernard, A. Blanchet, A. Bonhomme et al. (STEREO Collaboration), Sterile Neutrino Constraints from the STEREO Experiment with 66 Days of Reactor-On Data, Phys. Rev. Lett. 121, 161801 (2018).
[42] B. Armbruster, I. M. Blair, B. A. Bodmann, N. E. Booth, G. Drexlin et al. (KARMEN Collaboration), Upper limits for neutrino oscillations muon-anti-neutrino $\rightarrow$ electronanti-neutrino from muon decay at rest, Phys. Rev. D 65 , 112001 (2002).

[43] P. Adamson et al. (MiniBooNE and MINOS Collaborations), First Measurement of $\nu_{\mu}$ and $\nu_{e}$ Events in an OffAxis Horn-Focused Neutrino Beam, Phys. Rev. Lett. 102, 211801 (2009).

[44] P. Astier et al. (NOMAD Collaboration), Search for $\nu_{\mu} \rightarrow$ $\nu_{e}$ oscillations in the NOMAD experiment, Phys. Lett. B 570, 19 (2003).

[45] M. Dentler, A. Hernández-Cabezudo, J. Kopp, P. A. N. Machado, M. Maltoni, I. Martinez-Soler, and T. Schwetz, Updated global analysis of neutrino oscillations in the presence of eV-scale sterile neutrinos, J. High Energy Phys. 08 (2018) 010.

[46] S. Hannestad, I. Tamborra, and T. Tram, Thermalisation of light sterile neutrinos in the early universe, J. Cosmol. Astropart. Phys. 07 (2012) 025.

[47] M. Lattanzi and M. Gerbino, Status of neutrino properties and future prospects-Cosmological and astrophysical constraints, Front. Phys. 5, 70 (2018).

[48] A. M. Knee, D. Contreras, and D. Scott, Cosmological constraints on sterile neutrino oscillations from Planck, J. Cosmol. Astropart. Phys. 07 (2019) 039.

[49] J. M. Berryman, Constraining sterile neutrino cosmology with terrestrial oscillation experiments, Phys. Rev. D 100, 023540 (2019).

[50] S. Gariazzo, P. de Salas, and S. Pastor, Thermalisation of sterile neutrinos in the early universe in the $3+1$ scheme with full mixing matrix, J. Cosmol. Astropart. Phys. 07 (2019) 014.

[51] S. Hagstotz, P. F. de Salas, S. Gariazzo, M. Gerbino, M. Lattanzi, S. Vagnozzi, K. Freese, and S. Pastor, Bounds on light sterile neutrino mass and mixing from cosmology and laboratory searches, arXiv:2003.02289.

[52] M. Adams, F. Bezrukov, J. Elvin-Poole, J. J. Evans, P. Guzowski, B. O. Fearraigh, and S. Söldner-Rembold, Direct comparison of sterile neutrino constraints from cosmological data, $\nu_{e}$ disappearance data and $\nu_{\mu} \rightarrow \nu_{e}$ appearance data in a $3+1$ model, Eur. Phys. J. C 80, 758 (2020).

[53] R. Foot and R. R. Volkas, Neutrino physics and the mirror world: How exact parity symmetry explains the solar neutrino deficit, the atmospheric neutrino anomaly, and the lsnd experiment, Phys. Rev. D 52, 6595 (1995).

[54] G. Gelmini, S. Palomares-Ruiz, and S. Pascoli, Low Reheating Temperature and the Visible Sterile Neutrino, Phys. Rev. Lett. 93, 081302 (2004).

[55] C. M. Ho and R. J. Scherrer, Limits on mev dark matter from the effective number of neutrinos, Phys. Rev. D 87, 023505 (2013).

[56] S. Hannestad, R. S. Hansen, and T. Tram, How SelfInteractions can Reconcile Sterile Neutrinos with Cosmology, Phys. Rev. Lett. 112, 031802 (2014).

[57] M. Archidiacono, S. Hannestad, R. S. Hansen, and T. Tram, Cosmology with self-interacting sterile neutrinos and dark matter-A pseudoscalar model, Phys. Rev. D 91, 065021 (2015). 
[58] M. Archidiacono, S. Hannestad, R. S. Hansen, and T. Tram, Sterile neutrinos with pseudoscalar self-interactions and cosmology, Phys. Rev. D 93, 045004 (2016).

[59] B. Dasgupta and J. Kopp, Cosmologically Safe eV-Scale Sterile Neutrinos and Improved Dark Matter Structure, Phys. Rev. Lett. 112, 031803 (2014).

[60] N. Saviano, O. Pisanti, G. Mangano, and A. Mirizzi, Unveiling secret interactions among sterile neutrinos with big-bang nucleosynthesis, Phys. Rev. D 90, 113009 (2014).

[61] X. Chu, B. Dasgupta, and J. Kopp, Sterile neutrinos with secret interactions-Lasting friendship with cosmology, J. Cosmol. Astropart. Phys. 10 (2015) 011.

[62] X. Chu, B. Dasgupta, M. Dentler, J. Kopp, and N. Saviano, Sterile neutrinos with secret interactions-Cosmological discord? J. Cosmol. Astropart. Phys. 11 (2018) 049.

[63] N. Song, M. Gonzalez-Garcia, and J. Salvado, Cosmological constraints with self-interacting sterile neutrinos, J. Cosmol. Astropart. Phys. 10 (2018) 055.

[64] L. S. Miranda and S. Razzaque, Revisiting constraints on $3+1$ active-sterile neutrino mixing using IceCube data, J. High Energy Phys. 03 (2019) 203.

[65] M. G. Aartsen et al. (IceCube Collaboration), Searching for $\mathrm{eV}$-scale sterile neutrinos with eight years of atmospheric neutrinos at the IceCube neutrino telescope, Phys. Rev. D 102, 052009 (2020).

[66] M. G. Aartsen et al. (IceCube Collaboration), The IceCube neutrino observatory: Instrumentation and online systems, J. Instrum. 12, P03012 (2017).

[67] R. Abbasi et al. (IceCube Collaboration), The IceCube data acquisition system: Signal capture, digitization, and timestamping, Nucl. Instrum. Methods Phys. Res., Sect. A 601, 294 (2009).

[68] R. Abbasi et al. (IceCube Collaboration), The design and performance of IceCube DeepCore, Astropart. Phys. 35, 615 (2012).

[69] E. V. Bugaev, A. Misaki, V. A. Naumov, T. S. Sinegovskaya, S. I. Sinegovsky, and N. Takahashi, Atmospheric muon flux at sea level, underground and underwater, Phys. Rev. D 58, 054001 (1998).

[70] R. Gandhi, C. Quigg, M. H. Reno, and I. Sarcevic, Ultrahighenergy neutrino interactions, Astropart. Phys. 5, 81 (1996).

[71] N. E. Bramall, R. C. Bay, K. Woschnagg, R. A. Rohde, and P. B. Price, A deep high-resolution optical log of dust, ash, and stratigraphy in south pole glacial ice, Geophys. Res. Lett. 32, L21815 (2005).

[72] P. Askebjer et al., Optical properties of deep ice at the south pole: Absorption, Appl. Opt. 36, 4168 (1997).

[73] M. G. Aartsen et al. (IceCube Collaboration), Measurement of south pole ice transparency with the IceCube LED calibration system, Nucl. Instrum. Methods Phys. Res., Sect. A 711, 73 (2013).

[74] P. Lipari, Lepton spectra in the earth's atmosphere, Astropart. Phys. 1, 195 (1993).

[75] J. H. Koehne, K. Frantzen, M. Schmitz, T. Fuchs, W. Rhode, D. Chirkin, and J. Becker Tjus, PROPOSAL: A tool for propagation of charged leptons, Comput. Phys. Commun. 184, 2070 (2013).

[76] F. Halzen, Astroparticle physics with high energy neutrinos: From AMANDA to IceCube, Eur. Phys. J. C 46, 669 (2006).
[77] M. Aartsen et al. (IceCube Collaboration), Evidence for Astrophysical Muon Neutrinos from the Northern Sky with IceCube, Phys. Rev. Lett. 115, 081102 (2015).

[78] J. Ahrens et al. (AMANDA Collaboration), Muon track reconstruction and data selection techniques in AMANDA, Nucl. Instrum. Methods Phys. Res., Sect. A 524, 169 (2004).

[79] M. G. Aartsen et al. (IceCube Collaboration), Energy reconstruction methods in the IceCube neutrino telescope, J. Instrum. 9, P03009 (2014).

[80] D. Heck, J. Knapp, J. N. Capdevielle, G. Schatz, and T. Thouw, CORSIKA: A Monte Carlo code to simulate extensive air showers (1998).

[81] A. Fedynitch, R. Engel, T. K. Gaisser, F. Riehn, and T. Stanev, Calculation of conventional and prompt lepton fluxes at very high energy, EPJ Web Conf. 99, 08001 (2015).

[82] A. Fedynitch, J. Becker Tjus, and P. Desiati, Influence of hadronic interaction models and the cosmic ray spectrum on the high energy atmospheric muon and neutrino flux, Phys. Rev. D 86, 114024 (2012).

[83] F. Riehn, H. P. Dembinski, R. Engel, A. Fedynitch, T. K. Gaisser, and T. Stanev, The hadronic interaction model SIBYLL 2.3c and Feynman scaling, Proc. Sci., ICRC2017 (2018) 301 [arXiv:1709.07227].

[84] T. K. Gaisser, Spectrum of cosmic-ray nucleons, kaon production, and the atmospheric muon charge ratio, Astropart. Phys. 35, 801 (2012).

[85] A. M. Hillas, Cosmic rays: Recent progress and some current questions, in Conference on Cosmology, Galaxy Formation and Astro-Particle Physics on the Pathway to the SKA Oxford, England (2006) [arXiv:astro-ph/ 0607109].

[86] Jet Propulsion Laboratory, AIRS/AMSU/HSB Version 6 Level 3 Product User Guide, Version 1.2 (2014).

[87] A. Bhattacharya, R. Enberg, Y. S. Jeong, C. S. Kim, M. H. Reno, I. Sarcevic, and A. Stasto, Prompt atmospheric neutrino fluxes: perturbative QCD models and nuclear effects, J. High Energy Phys. 11 (2016) 167.

[88] A. Palladino and F. Vissani, The natural parameterization of cosmic neutrino oscillations, Eur. Phys. J. C 75, 433 (2015).

[89] C. A. Argüelles, T. Katori, and J. Salvado, New Physics in Astrophysical Neutrino Flavor, Phys. Rev. Lett. 115, 161303 (2015).

[90] M. Bustamante, J. F. Beacom, and W. Winter, Theoretically Palatable Flavor Combinations of Astrophysical Neutrinos, Phys. Rev. Lett. 115, 161302 (2015).

[91] A. Schneider, Characterization of the astrophysical diffuse neutrino flux with IceCube high-energy starting events, in 36th International Cosmic Ray Conference (ICRC 2019) Madison, Wisconsin, USA, (2019) [arXiv:1907.11266].

[92] C. A. Argüelles Delgado, J. Salvado, and C. N. Weaver, A Simple Quantum Integro-Differential Solver (SQuIDS), Comput. Phys. Commun. 196, 569 (2015).

[93] C. A. Argüelles, J. Salvado, and C. N. Weaver, nuSQuIDS, https://github.com/Arguelles/nuSQuIDS (2015).

[94] M. C. Gonzalez-Garcia, F. Halzen, and M. Maltoni, Physics reach of high-energy and high-statistics IceCube atmospheric neutrino data, Phys. Rev. D 71, 093010 (2005). 
[95] S. L. Glashow, Resonant scattering of antineutrinos, Phys. Rev. 118, 316 (1960).

[96] F. Halzen and D. Saltzberg, Tau-Neutrino Appearance with a 1000 Megaparsec Baseline, Phys. Rev. Lett. 81, 4305 (1998).

[97] A. Cooper-Sarkar, P. Mertsch, and S. Sarkar, The high energy neutrino cross-section in the Standard Model and its uncertainty, J. High Energy Phys. 08 (2011) 042.

[98] A. M. Dziewonski and D. L. Anderson, Preliminary reference earth model, Phys. Earth Planet. Inter. 25, 297 (1981).

[99] C. A. Argüelles, A. Schneider, and T. Yuan, A binned likelihood for stochastic models, J. High Energy Phys. 06 (2019) 030.

[100] S. S. Wilks, The large-sample distribution of the likelihood ratio for testing composite hypotheses, Ann. Math. Stat. 9, 60 (1938).

[101] G. J. Feldman and R. D. Cousins, A unified approach to the classical statistical analysis of small signals, Phys. Rev. D 57, 3873 (1998).

[102] U. von Toussaint, Bayesian inference in physics, Rev. Mod. Phys. 83, 943 (2011).

[103] F. Feroz, M. P. Hobson, and M. Bridges, MultiNest: An efficient and robust Bayesian inference tool for cosmology and particle physics, Mon. Not. R. Astron. Soc. 398, 1601 (2009).

[104] A. V. Karelin, S. V. Borisov, A. M. Galper, and S. A. Voronov, The proton and helium cosmic ray spectra from 50-GeV to 15-TeV, Astrophys. Space Sci. Trans. 7, 235 (2011).

[105] B. Bartoli et al. (ARGO-YBJ Collaboration), Cosmic ray proton plus helium energy spectrum measured by the ARGO-YBJ experiment in the energy range 3-300 TeV, Phys. Rev. D 91, 112017 (2015).

[106] Y.S. Yoon et al., Proton and helium spectra from the CREAM-III flight, Astrophys. J. 839, 5 (2017).

[107] R. Alfaro et al. (HAWC Collaboration), All-particle cosmic ray energy spectrum measured by the HAWC experiment from 10 to $500 \mathrm{TeV}$, Phys. Rev. D 96, 122001 (2017).

[108] G. D. Barr, S. Robbins, T. K. Gaisser, and T. Stanev, Uncertainties in atmospheric neutrino fluxes, Phys. Rev. D 74, 094009 (2006).

[109] F. Halzen, K. Igi, M. Ishida, and C. S. Kim, Total hadronic cross sections and $\pi^{\mp} \pi^{+}$scattering, Phys. Rev. D 85, 074020 (2012).

[110] M. G. Aartsen et al. (IceCube Collaboration), Evidence for high-energy extraterrestrial neutrinos at the IceCube detector, Science 342, 1242856 (2013).

[111] M. G. Aartsen et al. (IceCube Collaboration), Observation of High-Energy Astrophysical Neutrinos in Three Years of IceCube Data, Phys. Rev. Lett. 113, 101101 (2014).

[112] M. G. Aartsen et al. (IceCube Collaboration), Observation and characterization of a cosmic muon neutrino flux from the northern hemisphere using six years of IceCube data, Astrophys. J. 833, 3 (2016).

[113] M. G. Aartsen et al. (IceCube Collaboration), Measurements using the inelasticity distribution of multi-TeV neutrino interactions in IceCube, Phys. Rev. D 99, 032004 (2019).

[114] M. Aartsen et al. (IceCube Collaboration), Companion paper, Characteristics of the diffuse astrophysical electron and tau neutrino flux with six years of IceCube high energy cascade data, Phys. Rev. Lett. [arXiv:2001.09520 (to be published)].

[115] M. G. Aartsen et al. (IceCube Collaboration), Efficient propagation of systematic uncertainties from calibration to analysis with the SnowStorm method in IceCube, J. Cosmol. Astropart. Phys. 10 (2019) 048.

[116] A. Karle, Entwicklung eines neuartigen atmosphärischen Tscherenkovdetektors und Messungen an hochenergetischer Kosmischer Strahlung zwischen 15 und 1000 TeV, Ph.D. thesis, München University, 1994.

[117] M. Aartsen et al. (IceCube Collaboration), In-situ calibration of the single-photoelectron charge response of the IceCube photomultiplier tubes, J. Instrum. 15, P06032 (2020).

[118] M. G. Aartsen et al. (IceCube Collaboration), Measurement of Atmospheric Neutrino Oscillations at 6-56 GeV with IceCube DeepCore, Phys. Rev. Lett. 120, 071801 (2018).

[119] A. C. Vincent, C. A. Argüelles, and A. Kheirandish, High-energy neutrino attenuation in the Earth and its associated uncertainties, J. Cosmol. Astropart. Phys. 11 (2017) 012.

[120] A. Connolly, R. S. Thorne, and D. Waters, Calculation of high energy neutrino-nucleon cross sections and uncertainties using the MSTW parton distribution functions and implications for future experiments, Phys. Rev. D 83, 113009 (2011).

[121] C. A. Argüelles, New physics with atmospheric neutrinos, Ph.D. thesis, University of Wisconsin-Madison, 2015.

[122] B. J. Jones, Sterile neutrinos in cold climates, Ph.D. Thesis, MIT, 2015.

[123] G. Cheng et al. (MiniBooNE and SciBooNE Collaborations), Dual baseline search for muon antineutrino disappearance at $0.1 \mathrm{eV}^{2}<\Delta m^{2}<100 \mathrm{eV}^{2}$, Phys. Rev. D 86, 052009 (2012).

[124] G. H. Collin, C. Argüelles, J. M. Conrad, and M. H. Shaevitz, First Constraints on the Complete Neutrino Mixing Matrix with a Sterile Neutrino, Phys. Rev. Lett. 117, 221801 (2016).

[125] H. Jeffreys, The Theory of Probability (Oxford University Press, Oxford, 1998).

[126] S. N. Axani, Sterile neutrino searches at the IceCube neutrino observatory, Ph.D. thesis, MIT, 2019. 